\title{
29 Emotionale Belastungen in der Notfallmedizin
}

\author{
Karl-Heinz Henze und Gudrun Piechotta-Henze
}

\subsection{Einleitung}

Auf Notfälle müssen sich ärztliches und pflegendes Personal sowie die verschiedenen Berufsgruppen im Rettungswesen vorbereiten. Sie lernen während ihrer Ausbildung an der Universität bzw. an Hochschule und Fachschule, in Praxiseinsätzen innerhalb und außerhalb der Klinik, in Fort- und Weiterbildungen wie ein Mensch reanimiert wird, wie ein Defibrillator zu handhaben ist, welche Medikamente zu verabreichen sind etc. Ebenso wichtig ist, ethisch begründet zu handeln, das Selbstbestimmungsrecht des einzelnen Menschen in Notfallsituationen zu respektieren und - soweit dies möglich ist - eine gemeinsame Entscheidung mit dem Betroffenen und den Angehörigen herbeizuführen (Meyer u. Balck 2002).

Wie werden verschiedenste Notfallsituationen emotional ausgehalten und bewältigt? Diese und weitere Fragen haben wir drei Personen gestellt, die im Rahmen ihrer beruflichen Tätigkeit mit Notfällen konfrontiert sind: Silke Reinhardt28 (S.R.) ist Gesundheits- und Krankenpflegerin, sie arbeitet seit 22 Jahren auf einer interdisziplinären Notfallaufnahme eines Krankenhauses mit Maximalversorgung. Hartmut Schulz (H.S.) ist seit nunmehr 18 Jahren bei der Berufsfeuerwehr, er ist sowohl in Rettungs- als auch Feuerwehreinsätzen tätig. Klaus Heinrich (K.H.) ist Facharzt für Anästhesie. Nach Tätigkeiten im Operations- und Intensivbereich arbeitet er seit vier Jahren als Notarzt im Rettungswesen.

28 Die Angaben sind anonymisiert, die Namen Pseudonyme. 


\subsection{Emotionale Belastungen und ihre Auswirkungen}

Trotz aller Unterschiedlichkeit der verschiedenen Arbeits- und Aufgabenbereiche in den Feldern der Notfallversorgung sind Wechsel von Warte- bzw. Ruhezeiten und plötzlichen Arbeitseinsätzen, die dann höchste Konzentration und Kompetenz erfordern, charakteristisch. In den Zeiten vor Einsätzen sowie vor Aufnahmen von Patienten/-innen werden vorbereitende Aufgaben durchgeführt, etwa die Medikamente überprüft und aufgefüllt, die Geräte gewartet, es wird ferngesehen oder mit Kollegen und Kolleginnen gesprochen. ${ }^{29}$

In der Wartephase verspüren vor allem die weniger berufserfahrenen Akteure/-innen Anspannung, Unruhe, Unsicherheit und Versagensängste:

„Wenn man Anfänger ist, erschrickt man sich, ist ganz aufgeregt. Am Anfang habe ich immer gedacht, hoffentlich ist es etwas Leichtes, was ich gut behandeln kann. Später, wenn man viele Krankheitsbilder gesehen hat, ist man viel ruhiger." (K.H.)

Eine Belastungsspitze kann während der Anfahrt zu einem Notfall auftreten, weil viel Raum für Phantasien über die bevorstehende Situation besteht, und damit entsprechende Ängste ausgelöst werden.

Jenseits von relativ undramatischen Routineaufgaben können die Akteure/-innen extremen Stressoren ausgesetzt sein, sei es am Einsatzort (schwerer Verkehrsunfall, mehrere Schwerverletzte, Suizid, Tötungsdelikt) oder angesichts der Konfrontation mit einer schwerverletzten Person im Rahmen stationärer Notfallbehandlung. Sie sind mit einer Flut an eigenen Gefühlslagen und Befindlichkeiten konfrontiert, mit denen sie umgehen müssen, auch unter sozialethischen Aspekten. In besonderer Weise sind Notfälle gefürchtet, die Kinder, Jugendliche und junge Erwachsene betreffen:

„... Stirbt ein junger Mensch, etwa ein 20-Jähriger, ist man emotional sehr stark involviert, anders als bei einem alten Menschen, der sein Leben schon gelebt hat. Bei den jungen Menschen, könnte es immer dein Kind sein. Und man erlebt das als ungerecht und sinnlos. Ich frage mich dann immer:, Warum stirbt er? Er hat doch sein ganzes Leben noch vor sich' ... Dann steht man wirklich manchmal mit den Tränen in den Augen da."(S.R.)

„Ich werde nievergessen, als wir gerufen wurden und kamen in die Wohnung, in der die Mutter war und ihr totes Baby, das einen Plötzlichen Kindstod erlitten hatte ... Wenn Kinder, junge Leute betroffen sind, ist das schwer auszuhalten. Wenn du helfen willst, aber es geht nicht mehr." (H.S.)

Mit dem Tod von Menschen zu einem frühen Lebenszeitpunkt ist eine unmittelbare Trauerreaktion und Sinnkrise verbunden. Das betrifft nicht nur Eltern und Familienangehörige, sondern auch die Helfer und Helferinnen, die nicht mehr lebensrettend eingreifen konnten. Es resultieren Gefühle von Hilflosigkeit, Ohnmacht, womöglich auch von Versagen und Schuld. Während es sonst idealerweise durch eine Teilidentifikation gelingt, Anteil zu nehmen und gleichzeitig Arbeitsfähigkeit zu bewahren, ist das angesichts der unmittelbaren Konfrontation mit einem sterbenden und verstorbenen Kind kaum beizubehalten. In dem o.a. Zitat wird eine Identifizierung mit den Kindeseltern deutlich: „es könnte mein Kind sein“. Und doch verlangt ein solches

29 In der Notfallaufnahme, so S. R., hat sich die Zeit des Wartens allerdings zusehends verringert. Immer mehr Patienten/-innen müssen im Laufe des Tages und auch nachts versorgt werden. 
dramatisches Geschehen nach Handlungsfähigkeit, denn auch die Angehörigen bedürfen dringend der emotionalen Unterstützung.

Oftmals sind zeitgleich oder in kurzer zeitlicher Abfolge weitere Patienten/-innen sowie verunglückte Menschen zu versorgen. Im klinischen Bereich, der von zunehmendem „Patientendurchlauf“ und Arbeitsverdichtung geprägt ist, kann es dann dazu kommen, dass es ,... nur noch darum geht, in kürzester Zeit und mit aller Anstrengung das Leben zu retten. “ Die anderen Patienten/-innen und deren Angehörige werden „quasi nur noch aus den Augenwinkeln oder temporär gar nicht mehr wahrgenommen." (S.R.)

Die Selbststeuerungs-, Entscheidungs- und Bestimmungsfähigkeit kann in Situationen mit sehr hohem Belastungsgeschehen verloren gehen.

So fühlt sich ein Arzt zunächst handlungsfähig, erlebt sich vor Ort aber gelähmt angesichts einer Karambolage zwischen mehreren Autos, aus denen schwerverletzte Menschen zu bergen sind:

„Er hatte das Gefühl, weder denken noch reagieren zu können. Am Unfallort glaubte er, die Lage im Griff zu haben, doch binnen kürzester Zeit war er nicht mehr in der Lage, zu entscheiden, was getan werden muss. Wir konnten sehen, wie ihm alles entglitten ist." (H.S.)

Da das Rettungsteam seit langer Zeit zusammenarbeitet, kann ein anderer Helfer vor Ort umgehend die Aufgabenkoordination übernehmen, die Erstversorgung wird adäquat durchgeführt.

Idealerweise kann man eine solche Situation mit Gewinn - im Sinne eines berufsbezogenen Lernprozesses und einer emotionalen Entlastung - für alle Beteiligten analysieren. Problematisch wird es, wenn durch Kollegen/-innen bzw. Vorgesetzte daraus ein individuelles Versagen konstruiert wird, das beim Betroffenen Insuffizienzgefühle, Scham und Schamangst sowie Angst vor Diskreditierung hervorruft:

„Die Fehlerkultur ist zweifellos noch unbefriedigend. Es wird schnell fokussiert: ,Du bist derjenige, der nicht funktioniert hat.'... Besonders schwierig ist, wenn du einen Fehler zweimal machst. Unter Umständen wirst du dann in eine Ecke gestellt, und es wird gesagt: ,Du kannst das nicht.“" (H.S.)

Dadurch wird eine Botschaft transportiert, die das Zeigen und das Reden über Emotionen eher als Schwäche darstellt, weniger als Reflexionsfähigkeit, sodass beim nächsten Einsatz nicht mit mehr innerer Sicherheit gehandelt werden kann.

Gerade Berufsanfänger/-innen orientieren sich oft an den Einschätzungen und Bewertungen der erfahrenen Kräfte. Einerseits erfahren sie dadurch narzisstische Gratifikation, andererseits wird gleichsam normativ bestimmt, was als Belastung gilt und was der Belobigung wert ist. Ein „Hero“ sein zu wollen, bringt gerade jüngere Mitarbeiter/-innen dazu, die eigene emotionale Belastungsgrenze zu überschätzen und aktionistisch zu handeln (Salomon 2003).

\subsubsection{Traumatische Belastungen}

Bei Einsätzen in Extremsituationen ${ }^{30}$ sind die medizinischen Akteure/-innen bzw. das gesamte Team mit Leid, schwersten Verletzungen, Sterben und Tod konfrontiert; gegebenenfalls ist auch das eigene Leben oder das anderer Helfer und Helferinnen bedroht. Es ist dann möglich, dass neben akuten Belastungsreaktionen (ICD-10:

30 S. Salomon 2003, S. 246 (Fallbeispiel 5). 
F43.0) posttraumatische Belastungsreaktionen (ICD-10: F43.1) auftreten. Letztere stellen einen Oberbegriff dar, unter dem sowohl primäre Traumafolgen (akute Traumareaktion, posttraumatische Belastungsreaktion, posttraumatische Spätreaktion) als auch sekundäre Traumafolgen (chronische posttraumatische Störungen bis hin zu einer andauernden Persönlichkeitsveränderung nach Extremtraumatisierung bzw. eine Posttraumatische Persönlichkeitsstörung) auftreten (Ermann 1999: 102-125). Nach Teegen (2003 zit. in Niehörster 2006) sind PTBS-Symptomatiken - auf der Grundlage eines Vergleichs von fünf Berufsgruppen - in besonderer Weise bei Intensivpflege und Rettungsdienst zu finden. Niehörster (2006) selbst gibt eine Prävalenz von PTBS-Symptomen bei $7 \%$ der Rettungsassistenten in Deutschland an.

Ein „psychisches Trauma stellt ... eine plötzliche Diskontinuität des körperlichen und psychischen Erlebens dar. Ein nicht erwartetes Eindringen äußerer Kräfte in die psychische Organisation. .... ein Zustand der Überwältigung des Ich durch innere und äußere Kräfte, die es hilflos machen hinsichtlich seiner unmittelbaren Anstrengungen zur Adaption“ (Wöller 2009: 11). In der Notfallmedizin dürfte überwiegend eine Gefährdung durch die sogenannten Typ-I-Traumen bestehen, d.h. durch eine unerwartete schockhafte Erfahrung. In dem Zusammenhang ist von besonderer Bedeutung, dass der Erhalt oder die Wiederherstellung von Selbstregulierungs-, Selbststeuerungs- und Selbstkontrollfähigkeit vorübergehend sein kann und erst Tage oder Wochen später die Folgen des Traumas offenbar werden können.

Charakteristisch kann auch eine subtraumatische chronische Überbelastung sein infolge belastender Konfrontationen, die jeweils zwar in unterschiedlichen Formen verarbeitet werden, aber letztlich durch ihre Permanenz und Sequenzialität schwächen und Symptomatiken hervorrufen können. Infolge dessen kann sich beispielsweise eine Suchtproblematik zeigen: „Es sind schon einige mit Alkoholproblemen nach Hause gegangen, weil sie es nicht mehr in den Griff gekriegt haben." (H.S.)

Bei traumatischen Erfahrungen im engeren Sinn resultieren in unterschiedlicher Ausprägung, Häufigkeit und Dauer ein fehlendes Sicherheitsgefühl, Hilflosigkeit, affektive Überflutung, gegebenenfalls völlige Erschöpfung, Leereempfinden, Ängste, chronische Übererregung (hyperarousal) oder reduzierte Empfindungsfähigkeit bzw. Abstumpfung, chronische Selbstvorwürfe, Depressivität, somatoforme Beschwerden (vgl. Ermann 1999; Wöller 2009). Durch sogenannte Trigger kann das traumatische Geschehen oder Teile davon jederzeit erneut ausgelöst werden. Dann finden „Intrusionen“ bzw. „Flashbacks“ statt, welche die Betroffenen erneut mit dem erlebten traumatischen Geschehen affektiv überfluten. Das Trauma wird gleichsam erneut durchlaufen, und es kommt immer wieder zum Zusammenbruch der Ich-Regulation bzw. zu einer psychischen Dekompensation. Das Trauma bleibt ein „nicht assimilierbarer Fremdkörper“ (Wöller 2009: 14).

\subsection{Umgang mit emotionalen Belastungen}

In allen Arbeits- und Lebensbereichen gibt es eine Palette von individuellen Bewältigungsstrategien und -mustern. Die typischen unbewussten Abwehrmodi (z.B. Verleugnung, Isolierung vom Affekt, furor therapeuticus im Sinne von Balint), können einerseits eine psychische Überbelastung erträglich machen, andererseits das bewusste Bearbeiten einer affektiven Unlust- oder Überflutungsreaktion verhindern: 
„Am Anfang meiner Tätigkeit im Rettungsdienst hatten wir diesen Fall von einem Plötzlichen Kindstod in einer Familie. Mir hatte man organisatorische und administrative Aufgaben zugeteilt. Die betroffene Mutter rief immer wieder: ,Warum trifft es immer mich?' Da musste ich rausgehen und bekomme noch heute eine Gänsehaut. Ein Kollegevon mir hat sich dann rührend, mit großer Empathie um die Mutter gekümmert.... Da ist mir bewusst geworden, dass man bestimmte Aufgaben als Einsatzkraft gerne anderen überlässt und daraufverweist, dass es die machen sollen, die dafür ausgebildet sind. Aber in der Situation hat sich auch gezeigt, dass man immer ausloten muss, wer kann was gut leisten. Für mich ist das bis heute ein überzeugendes Modell ... Das funktioniert nicht immer, doch wenn es funktioniert, dann kann man auch gut aus der Situation rausgehen." (H.S.)

In dem o.a. Beispiel weicht ein Akteur in einer zutiefst existentiellen Situation, einer Konfrontation mit einer verzweifelten Mutter, aus, indem er sich innerlich distanziert und die Aufgabe, Kontakt aufzunehmen, delegiert. Jenseits einer solchen defizitorientierten Perspektive geschieht etwas Wichtiges: Der Kollege fungiert als Modell, das exemplarisch zeigt,

1. welche Bedeutung Empathie und Einfühlungsvermögen für die Betroffenen haben,

2. dass derlei belastende Situationen bewältigt werden können und

3. dass man aus einer hochdramatischen Lage sogar bereichert hervorgehen kann.

Abgesehen von Abwehrmechanismen kann man die eigentlichen Bewältigungsmechanismen in intrapsychische Verarbeitungsmodi, instrumentelle Aktivitäten und soziale Unterstützungssysteme kategorisieren. Eine adäquate Verarbeitung gelingt zumeist dann,

- wenn man das Leben als sinnhaft und verstehbar erlebt,

- eine Fähigkeit besteht, die eigenen Emotionen artikulieren zu können,

- eine hohe psychische Flexibilität vorhanden ist, sich auf neue Situationen einzustellen,

- Bereitschaft zu einer aktiven Auseinandersetzung besteht und

- ein sozial unterstützendes Umfeld (Familie, Freundeskreis, Kollegen und Kolleginnen) existiert.

Konkret wurden als hilfreich beschrieben:

\section{Reflexionsvermögen und Bereitschaft zum lebenslangen (fachlichen) Lernen}

„Auf sich selbst schauen und gucken, was kann ich und was kann ich nicht. ... Was setzt mich massiv unter Druck und was weniger. Wenn man stagniert und immer die Dinge abspult, die man vor Jahren gelernt hat, das ist eine fatale Sache. Wichtig ist, sich auf jede Situation neu einzulassen und das heißt auch: Wir haben mit Menschen zu tun und jeder Mensch ist anders." (K.H.)

\section{Fachkompetenz und emotionale Kompetenz, die Sicherheit, Einfühlungsvermögen und Empathie ermöglichen}

„Die Patienten (am Unfallort) sind ja ganz unterschiedlich: Die einen sind ganz ruhig, die anderen schreien, lassen sich nicht beruhigen. In jedem Fall begebe ich mich auf Augenhöhe mit ihnen, meist liegen sie ja irgendwo. Ich knie mich hin, sodass ich nicht von oben herab 
spreche. Ich frage, was passiert ist und ob sie Schmerzen haben. Ich beruhige sie, überhaupt versuche ich, viel mit innen zu sprechen. Es gibt eine Aufgabenteilung, die Rettungsassistenten machen die Aufgaben rundum, sodass ich mich um die Patienten kümmern kann. Kontinuierlich erkläre ich, was wir jetzt machen. Die meisten bekommen nicht einmal die Hälfte mit, da sie so geschockt sind. Aber ich versuche mich immer in die Lage zu versetzen, wenn ich da liegen würde. Und für mich wäre wichtig, dass sich jemand um mich kümmert und mir erklärt, was aktuell gemacht wird. ... Wenn sie sich gar nicht durch Worte beruhigen lassen, dann muss man ihnen auch etwas spritzen, damit sie ein wenig „runterkommen“. (K.H.)

\section{Entlastung durch ein Primat des Handelns und limitierten Kontakt}

„In der Akutsituation muss man handeln! ... Man hat nur wenig Zeit und gleichzeitig ist so viel in der Situation zu tun, dass man gar nicht an die eigenen Gefühle denkt. ... Ich nehme wenig mit nach Hause, was vor allem darauf zurückzuführen ist, dass man die Patienten nur kurze Zeit kennt und das familiäre Umfeld nicht kennt." (S.R.)

\section{Kollegialer, vertrauensvoller Austausch, durch den auch eigene krisenhafte Befindlichkeiten bzw. Ängste reflektiert und gemildert werden}

„Wichtig ist, dass man immer wieder mit den Kollegen über das Geschehen spricht, wie die Menschen am Unfallort versorgt wurden etc., und auch darüber, was womöglich nicht so gut war und beim nächsten Mal anders gemacht werden sollte. Im Freundeskreis und innerhalb der Familie erzähle ich nichts mehr über meine Arbeit, da sie das nicht verstehen können. Für Außenstehende klingt das alles hochdramatisch. Viele wollen auch gar nichts davon hören." (K.H.)

\section{Unterstützende und wertschätzende Haltung seitens der Vorgesetzten sowie eine positive Fehlerkultur}

„Wichtig ist, dass die Leitungen gegebenenfalls rasch eingreifen und es schaffen, dass man sich nicht gegenseitig Vorwürfe macht. Wir haben u.a. eine sehr engagierte Pflegedienstleitung, die sogar nachts kommt und versucht, personelle Unterstützung, etwa studentische Aushilfskräfte, zu bekommen. Überhaupt ist wichtig, dass ein Klima der Wertschätzung herrscht. Obwohl einen der Arbeitsaufwand aggressiv werden lässt und Gefühle von Überforderung einsetzen, darf dies nicht auf den Einzelnen projiziert werden. Stattdessen müssen die Strukturen als Ursache im Blick bleiben." (S.R.)

„Die täglichen Frühbesprechungen sind echt gut. Es wird nie gesagt: ,Gott, wie konntest Du denn?' Unser Oberarzt sagt häufig: ,Du warst vor Ort, du hast viel Berufserfahrung, du wirst das richtig entschieden haben!' Das finde ich sehr gut! Es nimmt einem auch die Angst vor Fehlentscheidungen. Es gibt einem Sicherheit, dass man da nicht fertig gemacht wird. Man lernt nachzudenken und gut zu argumentieren, warum man dies oder das so gemacht hat und als richtig erachtet hat." (K.H.)

\section{Seelsorgerische und psychologische Unterstützung}

„Wenn du Hilflosigkeit bei dir selber oder bei den Kollegen erlebst und dann kommt einer von der Notfallseelsorge, also jemand, der nachfragt und sich kümmert, dann ist das sehr entlastend" (H.S.) 


\title{
Interdisziplinäre Zusammenarbeit
}

\begin{abstract}
„Bei uns ist die Zusammenarbeit von ärztlichem und pflegendem Personal gut - und tut auch gut! Alle wissen, dass wir zusammenarbeiten müssen. Jeder weiß, wenn wir gegeneinander arbeiten, können wir einpacken. Jeder weiß, dass man den anderen braucht!“ (S.R.)
\end{abstract}

\section{Freizeit zur Wahrung einer psychosozialen Balance}

„Der Freizeitausgleich ist sehr wichtig! Manchmal mache ich zu Hause sogar das Telefon aus, sodass ich wirklich ,runter komme.' Ich habe bewusst auch meine Arbeitszeit reduziert, damit ich ausreichend Privatleben habe und Regenerationsphasen." (S.R.)

\subsection{Weitere Überlegungen und Folgerungen}

In den letzten Jahren wird aus einer eher anthropologischen Perspektive das der Notfallmedizin zugrundeliegende Menschenbild thematisiert (Salomon 2003). Unter anderem wird geschlussfolgert, dass alle im Rettungsdienst Tätigen, mit Subjekten zu tun haben, auch wenn sie diese phasenweise - im Dienste der Lebensrettung gleichsam objekthaft behandeln müssen. Wenn man einige der o.a. Zitate unter diesem Aspekt liest, wird eine entsprechende Grundhaltung der Akteure/-innen sichtbar: Sie bemühen sich bei der Ausübung ihrer beruflichen Rolle, auf ein Subjekt einzugehen, ihr Handeln kontinuierlich zu überprüfen und ihr Erleben im Diskurs zu reflektieren, um die existentielle Not und das Leiden der schwer verunfallten oder erkrankten Menschen auszuhalten und nicht durch unbewusste Abwehrprozesse ${ }^{31}$ die eigenen Ängste zu bändigen. Eine Umgestaltung des eigenen Rollenverständnisses hat auch Implikationen für die Helfer/-innen selbst. Salomon hat darauf hingewiesen, dass die psychische Betreuung für Helfer und Helferinnen „einen immer höheren Stellenwert gewinnt, nachdem zugelassen wird, dass auch stark anmutende Helfer Menschen sind, die Belastungsgrenzen haben und Hilfe brauchen." (Salomon 2003: 245)

Ein weiterer Aspekt ist von besonderer Bedeutung: In existentiellen Bedrohungen, die in der Konfrontation mit Leiden, schwersten körperlichen Verletzungen, Schmerzen, Sterben und Tod eines anderen Menschen, resultieren können, besteht die Gefahr, dass eine bis dahin ausreichende Sinnordnung defizitär wird und der eigene Lebenssinn in eine - nicht selten latent bleibende - existentielle Krise gerät. Erst wenn Sterben und Tod als unabänderliches Wesensmerkmal des Lebens immer wieder sinnhaft legitimiert werden können, ist es auch möglich, die Sinnhaftigkeit des eigenen Handelns wieder herzustellen (Berger 1973).

In der Aus- und Weiterbildung ist die Vermittlung eines solchen Rollenverständnisses, das Erlernen einer fallbezogenen Diskurs- und Fehlerkultur, die Förderung von Teamfähigkeit sowie die Fähigkeit sich mit den eigenen Affekten bewusst auseinandersetzen zu können, um so die Verstehensgrenzen besser ausloten zu können, bedeutsam (Bein u. Graf 2012). Wichtig ist zudem das Erlernen einer hohen Ambigui-

31 Sind die Ziele ausschließlich auf „Lebensrettung“ und „Krankheitsbekämpfung“ ausgerichtet, kann es angesichts des Sterbens, das gleichsam als Niederlage empfunden wird, zu Gefühlen von drohendem Misserfolg, Hilflosigkeit und Ohnmacht kommen, die durch hektische Aktivität kompensiert werden. 
tätstoleranz. Gerade in der Notfallmedizin, die von Entscheidungszwängen und Prioritätensetzung geprägt ist, gibt es unauflösbare Entscheidungskonflikte. In einer drängenden Situation müssen schnell Entscheidungen gefällt werden - die oft nicht widerspruchsfrei sind.

\section{Literatur}

Bein T, Graf BM (2012) Ethische Fallbesprechung in der Intensivmedizin. Der Anaesthesist 1: 6-13

Berger PL (1973) Zur Dialektik von Religion und Gesellschaft. Elemente einer soziologischen Theorie. Fischer, Frankfurt am Main

Ermann M (1999) Psychotherapeutische und psychosomatische Medizin. 3. Ausfl. Kohlhammer, Stuttgart, Berlin, Köln

Meyer W, Balck F (2002) Notärzte im Umgang mit psychisch belastenden Einsatzstrategien. Bewältigungsstrategien und notärztliche Persönlichkeiten. Notfall \& Rettungsmedizin 2: 89-95

Niehörster G (2006) Auswirkungen von psychischen Belastungen auf die Gesundheit von Rettungsdienstmitarbeitern. http://www2.medizin.uni-greifswald.de/intensiv/fileadmin/user_upload/lehre/qsb_notfallmedizin/ vorlesungen_qsb3/Vortrag_VL_Notfallmedizin.pdf (Zugriff am 12.06.2015)

Salomon F (2003) Das Menschenbild als Hintergrund notfallmedizinischer Entscheidungen. Notfall \& Rettungsmedizin 6: 242-246

Wallner I (2009) Organisation medizinischer Entscheidung am Lebensende. Intensivmedizin 47: 49-54

Wöller W (2009) Trauma und Persönlichkeitsstörungen. Schattauer, Stuttgart, 1. unveränd. Nachdruck

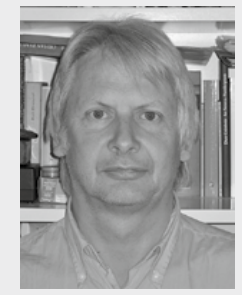

\section{Dr. disc. pol. Karl-Heinz Henze}

Wissenschaftlicher Angestellter im Institut für Medizinische Psychologie und Medizinische Soziologie der Georg-August-Universität Göttingen sowie Psychotherapeut in eigener Praxis; Forschungsschwerpunkte: Psychosoziale Thematiken in den Feldern „Chronische Krankheit" sowie „Sterben und Tod“; gemeinsame Entscheidungsfindung (Bilanzierungsdialog).

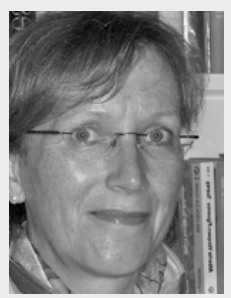

\section{Prof. Dr. Gudrun Piechotta-Henze}

Krankenschwester, Soziologin (M.A.), Professorin für Pflegewissenschaft an der Alice Salomon Hochschule Berlin in den Studiengängen „Gesundheits-und Pflegemanagement" (B.SC.) \& „Management und Qualitätsentwicklung im Gesundheitswesen" (M.Sc.); Arbeits- und Forschungsschwerpunkte: Begleitung und Pflege von Menschen mit Demenz, Beratung im Gesundheitsbereich, Emotionen in der Pflege, kultursensible Pflege, Menschenrechte und Menschenwürde, historische Pflegeforschung. 